\title{
Perfil do administrador de clínica of talmológica na Grande São Paulo
}

\author{
The profileof the ophthalmic clinic manager in São Paulocity and neighboring towns
}

\author{
Jorge Wilson Nogueira Neves ${ }^{1}$ \\ Marcio Boaventura Maia ${ }^{2}$
}

\footnotetext{
${ }^{1}$ Mestrando do Mestrado Profissionalizante da Universidade Federal de São Paulo - UNIFESP.

${ }^{2}$ Doutor em Oftalmologia pela Universidade Federal de São Paulo - UNIFESP.

Endereço para correspondência: Rua Dr.Alcides Ricardini Neves, 12/318 - São Paulo (SP)

CEP 04575-050 - E-mail: jwilson@uol.com.br

Recebido para análise em 01.02.2002

Versão revisada recebida em 14.05.2003

Aprovação em 02.06.2003

Nota Editorial: Pela análise deste trabalho e por sua anuência na divulgação desta nota, agradecemos aos Drs. Wilmar Roberto Silvino e Paulo Gilberto Jorge Fadel.
}

\begin{tabular}{|c|}
\hline RESUMO \\
\hline $\begin{array}{l}\text { Objetivo: Determinar o perfil do administrador de clínica oftalmológica na } \\
\text { Grande São Paulo. Métodos: Foram encaminhados questionários para clíni- } \\
\text { cas oftalmológicas sorteadas aleatoriamente. Variáveis analisadas: sexo, } \\
\text { idade, escolaridade, cursos de graduação e pós-graduação, participação na } \\
\text { sociedade, carga horária efetiva, visão de carreira, e sistemática de remune- } \\
\text { ração e número de consultas/mês da clínica. Resultados: Cinqüenta e cinco } \\
\text { por cento eramhomens, } 60 \% \text { tinham de } 30 \text { a } 59 \text { anos, para } 60 \% \text { era suaprimeira } \\
\text { experiência na área e } 56 \% \text { administravam clínicas com mais de } 1000 \text { consul- } \\
\text { tas/mês. Entre os que tinham formação universitária (70\%), 56\% concluíram } \\
\text { curso de Administração de Empresas. Metade dos entrevistados tinham } \\
\text { pós-graduação, sendo os cursos preferidos: Administração Hospitalar } \\
\text { (40\%) e MBA(30\%). Somente } 10 \% \text { eram sócios da clínica. Trabalham acima } \\
\text { de40 horas/semana } 75 \% \text { dosprofissionais. Atualmente } 60 \% \text { não fazem curso } \\
\text { na área e } 56 \% \text { acham que o incentivoà carreira deve vir da clínica. Benefícios: } \\
75 \% \text { dos profissionais têm planos de saúde e/ou carteira assinada. Remune- } \\
\text { ração: salário comparticipaçãono faturamento (48\%) e salário fixo (42\%). } \\
\text { Conclusões: Neste estudo, encontramos a profissão de administrador de } \\
\text { clínicas estruturando-se com profissionais de ambos os sexos, formação em } \\
\text { Administração de Empresas, alguns com pós-graduação na área, carga } \\
\text { horária acima de } 40 \text { h semanais, acreditando que a clínica deve investir em } \\
\text { seu aperfeiçoamento. Fazem-se necessários outros estudos que contribuam } \\
\text { para melhor conhecimento deste profissional. }\end{array}$ \\
\hline
\end{tabular}

Descritores: Administração da prática médica/tendências; Administração de consultório/ tendências; Estudo de casos organizacionais; Diretores médicos; Gerenciamento da prática profissional/tendências; Prática profissional; Otimização

\section{INTRODUÇ̃̃̃O}

Recentemente, médicos têm se voltado para adquirir conhecimentos administrativos, ou então consegui-los através de alguém que os ajude profissionalmente nessa área. Essa ajuda vem da necessidade de alguém, médico ou não, se dedicar aos aspectos logísticos da atividade médica, cada vez mais complexa. E o que faz um administrador? Segundo Fayol, (1968) revisando neoclássicos como: Drucker, Koontz \& O’Donnell, Newman etc., cumpre ao administrador planejar, organizar, dirigir e controlar as organizações ${ }^{(1)}$.

No desempenho de suas funções, o administrador representa os seguintes papéis: relação (como líder, contato e representante oficial), comunicador (porta-voz, disseminador e monitorizador de informações) e executivo (como empreendedor, alocador de recursos, gerenciador de problemas e negociador) $)^{(2)}$.

Além dessas funções, acrescente-se a essas diferentes visões das funções administrativas a de educador, tendo em vista o compromisso desse 
profissional com projetos de transformação social, para os quais necessitará favorecer o desenvolvimento integral das pessoas com quem trabalha ${ }^{(3)}$.

Ao administrador cabe planejar, organizar, supervisionar os serviços técnico-administrativos, utilizando recursos financeiros e materiais, estabelecendo rotinas para assegurar a eficácia e qualidade dos serviços prestados ${ }^{(4)}$. O contínuo crescimento e a complexidade dos procedimentos médicos oftalmológicos têm levado os médicos oftalmologistas a se agruparem em sociedades ou cooperativas. Por outro lado, a disputa de novos mercados e o surgimento de novos conceitos e valores levaram à reestruturação da cultura organizacional vigente. Antes, erros administrativos, falhas e ineficiência permaneciam escondidos nos seios organizacionais e eram transferidas para os preços de produtos e serviços. O consumidor (paciente ou serviço de saúde) absorvia esses custos sem grande resistência. Hoje, a busca pela qualidade exige de todos, inclusive dos oftalmologistas, profunda revisão desses conceitos e valores, para sobreviver e crescer no meio dessas mudanças.

Com tudo isto, surge o interesse pela pessoa do administrador da organização de saúde.

\section{OBJETIVOS}

Esse trabalho tem como objetivo estudar e determinar o perfil do administrador de clínica oftalmológica na Grande São
Paulo. Tentaremos descobrir se os oftalmologistas, em suas clínicas, administram pessoalmente sua clínica ou se preferem trazer um profissional de outra área para atender essa exigência, enquanto se dedicam integralmente aos seus pacientes. Tentaremos também abordar alguns aspectos das necessidades dos profissionais que atualmente trabalham como administradores de clínicas oftalmológicas e sua visão de carreira profissional.

\section{MÉTODOS}

Clínicas oftalmológicas da Grande São Paulo foram selecionadas no segundo semestre de 2002 a partir de levantamento feito em listas de divulgação telefônica e de convênios. Foram enviados questionários (Anexo 1) após contato com sua administração.

Foram analisadas sexo, idade, escolaridade, realização de cursos de graduação e pós-graduação, a visão do administrador em relação à sua própria carreira e se participa ou pretende participar de algum curso na área. Analisamos também o número efetivo de horas semanais dedicadas à função, a participação na sociedade ou qual era sua sistemática de remuneração e o porte da clínica (usando o número médio de consultas/mês).

As informações recebidas foram tabuladas e analisadas no programa Epi-Info6, que forneceu a estatística descritiva dos dados.

\section{Perfil do administrador de Clínica Oftalmológica na Grande São Paulo}

Ficha:

Nome

Sexo

Idade

Instituição:

Graduação: Sim ( ) Não( ) - Méd ( ) Adm ( ) Mkt ( ) Publ ( ) Direito ( )
Pós-graduação Sim ( ) Não ( )
\[ \text { AdmHosp ( ) Mkt ( ) Publ. ( ) MBA ( ) Direito ( ) RH ( ) } \]
Sócio: Sim ( ) Não ( )

Consultas/mês Horas/sem Freqüência de reunião /sócios Benefícios: Sim ( ) Não ( ) Carteira Assinada: Sim ( ) Não ( ) Plano de saúde: Sim ( ) Não( ) Seguros: Sim( ) Não ( ) Primeira experiência: Sim ( ) Não ( ) 


\section{RESULTADOS}

Na tabela 1 encontramos o resultado da resposta dada aos questionários enviados.

Analisados os 20 questionários devolvidos, foram estudados o sexo e as faixas etárias dos administradores, conforme a tabela 2 e gráfico 1.

No estudo específico da profissão, foram avaliados: a experiência anterior na área, a existência de curso superior e os tipos de cursos realizados. Estas distribuições aparecem nas tabelas 3, 4, 5 e 6.

Entre os profissionais sem formação superior, os homens aparecem com freqüência superior às mulheres (Tabela 7).

A pós-graduação faz parte da formação de vários administradores. As tabelas 8, 9 e 10 apresentam o estudo desta variável quanto à sua realização, sexo e área escolhida.

Os profissionais, independente de seu nível de formação atual, demonstraram interesse em diversas áreas de aperfeiçoamento que viriam suprir suas necessidades. A tabela 11 mostra esta distribuição.

As tabelas 12 e 13 avaliam a participação do administrador como sócio da clínica, sendo médico ou não.

Para caracterizar o porte da clínica, levantamos o número de consultas/mês, apresentados na tabela 14 .

\begin{tabular}{|lcr|}
\hline \multicolumn{3}{|c|}{$\begin{array}{c}\text { Tabela 1. Distribuição dos resultados de respostas a questionários } \\
\text { enviados às clínicas oftalmológicas. São Paulo 2002 }\end{array}$} \\
Número de questionários & Freqüência & $\%$ \\
Respondidos & 20 & 59 \\
Não retornados & 12 & 35 \\
Recusa em responder & 2 & 6 \\
Total & 34 & 100 \\
\hline
\end{tabular}

\begin{tabular}{|lcc|}
\hline \multicolumn{3}{|c|}{ Tabela 2. Distribuição dos administradores de clínicas oftalmoló- } \\
gicas por sexo. São Paulo 2002
\end{tabular}

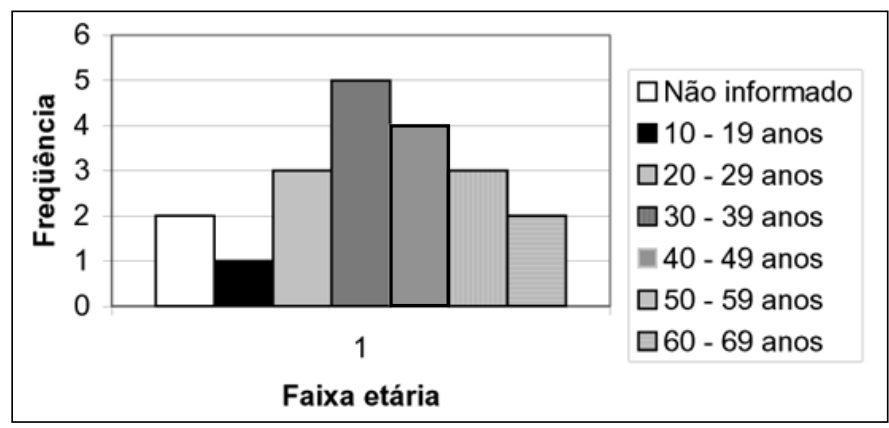

Gráfico 1 - Distribuição dos administradores de clínicas oftalmológicas, por faixa etária. São Paulo 2002

\begin{tabular}{|c|c|c|c|}
\hline $1^{a}$ experiência & Freqüência & $\%$ & $\%$ Acumulado \\
\hline Sim & 12 & 60 & 60 \\
\hline Não & 7 & 35 & 95 \\
\hline Não respondeu & 1 & 5 & 100 \\
\hline Total & 20 & 100 & \\
\hline
\end{tabular}

\begin{tabular}{lcc|}
$\begin{array}{l}\text { Tabela 4. Distribuição dos administradores de clínicas oftalmoló- } \\
\text { gicas, segundo o nível de formação. São Paulo 2002 }\end{array}$ \\
Nível superior & Freqüência & $\%$ \\
Sim & 14 & 70 \\
Não & 6 & 30 \\
Total & 20 & 100 \\
\hline
\end{tabular}

\begin{tabular}{|lcc|}
\hline \multicolumn{2}{|c|}{$\begin{array}{c}\text { Tabela 5. Distribuição de médicos que respondem como adminis- } \\
\text { tradores de clínicas. São Paulo } \mathbf{2 0 0 2}\end{array}$} \\
Médicos na administração & Freqüência & $\%$ \\
Sim & 2 & 10 \\
Não & 18 & 90 \\
Total & 20 & 100 \\
\hline
\end{tabular}

\begin{tabular}{lcc|}
$\begin{array}{l}\text { Tabela 6. Distribuição quanto à área de graduação, de administra- } \\
\text { dores de clínicas oftalmológicas. São Paulo 2002 }\end{array}$ \\
$\begin{array}{lcc}\text { Curso superior } & \text { Freqüência } & \% \\
\text { Medicina } & 2 & 12 \\
\text { Administração } & 9 & 56 \\
\text { Publicidade } & 1 & 7 \\
\text { Direito } & 1 & 7 \\
\text { Outros } & 3 & 18 \\
\text { Total } & 16^{*} & 100 \\
\text { * Aqui foi considerado o fato de que 2 administradores possuíam 2 cursos } \\
\text { em nível de graduação }\end{array}$ \\
\hline
\end{tabular}

\begin{tabular}{|c|c|c|}
\hline \multicolumn{3}{|c|}{$\begin{array}{l}\text { Tabela 7. Distribuição dos administradores de clínicas oftalmológica } \\
\text { sem curso de graduação, quanto ao sexo. São Paulo } 2002\end{array}$} \\
\hline Sem graduação & Freqüência & $\%$ \\
\hline Masculino & 4 & 66 \\
\hline Feminino & 2 & 34 \\
\hline Total & 6 & 100 \\
\hline
\end{tabular}

\begin{tabular}{|lcc|}
$\begin{array}{l}\text { Tabela 8. Distribuição segundo a realização de pós-graduação, da } \\
\text { freqüência de administradores de clínicas oftalmológicas. São } \\
\text { Paulo }\end{array}$ & $\begin{array}{l}\text { 2002 } \\
\text { Pós-graduação }\end{array}$ & Freqüência \\
Sim & 10 & $\%$ \\
Não & 10 & 50 \\
Total & 20 & 50 \\
\hline
\end{tabular}


No relacionamento da clínica com o profissional foram avaliados a carga horária trabalhada, a política de benefícios, o sistema de remuneração adotado e a freqüência de reuniões com os sócios. Tais distribuições aparecem nas tabelas de 15 a 19.

No estudo do desenvolvimento profissional do administrador, foram avaliadas suas expectativas, segundo sua visão de responsabilidade de investimento na carreira, áreas de estudo necessárias e participação atual em algum curso relacionado. As tabelas 20 e 21 mostram estas distribuições.

\section{DISCUSSÃO}

Dos 34 questionários enviados, 2 clínicas recusaram-se a fornecer qualquer informação e 12 clínicas não retornaram no prazo estipulado para a pesquisa. Desse modo, nosso índice de retorno foi de $59 \%$. Acreditamos que pesquisa deste tipo é um fato novo e que, com o tempo, haverá adesão mais consistente a esse tipo de pesquisa, o que talvez explique os questionários não retornados e os recusados. O conhecimento dos resultados deste trabalho e o aperfeiçoamento deste instrumento serão elementos essenciais para diminuir o número de não-respostas.

Houve equilíbrio na freqüência de ambos os sexos (55\% homens e $45 \%$ mulheres). Os administradores têm predominantemente (60\%) entre 30 e 59 anos de idade. A década de maior freqüência foi a de 30 a 39 anos. Em pesquisa com administradores hospitalares no Brasil encontrou-se 62,5\% dos mesmos abaixo dos 50 anos de idade ${ }^{(5)}$. Em pesquisa com executivos médicos, nos Estados Unidos (EUA), detectou-se média de idade de 54 anos $^{(6)}$.

A maioria (60\%) destes profissionais está em sua primeira experiência na área, independente de ter ou não experiências administrativas em outras áreas.

Por ser uma especialidade nova, toda a bagagem administrativa do profissional tem de ser desenvolvida ou adaptada no próprio exercício da função.

Os administradores têm predominantemente entre profissionais de formação universitária (70\%) e os administradores de empresa correspondem a 56\% da amostra, seguido dos médicos (12\%). Dois administradores possuíam mais de um curso em nível de graduação.

É relativamente baixo o número de profissionais médicos que respondem diretamente pela administração; apenas 2 (10\%) na amostra. Encontrou-se 14 médicos que administravam hospitais ( $25 \%$ da amostra) e, desses, 3 não tinham especialização em administração hospitalar ${ }^{(5)}$.

Apenas 6 administradores (30\%) não possuem curso de graduação. Mezomo em 1987, em pesquisas com administradores de instituições de saúde encontrou um percentual de $5 \%$ de profissionais sem curso superior ${ }^{(5)}$.

O número de homens sem graduação é o dobro do de mulheres.

Entre os profissionais da amostra, 50\% têm curso de pósgraduação. Há ligeiro predomínio de pós-graduação entre os administradores de sexo masculino (60\%).

\begin{tabular}{|lcc|}
\hline $\begin{array}{c}\text { Tabela 9. Distribuição, por sexo, dos administradores de clínicas } \\
\text { oftalmológicas com pós-graduação. São Paulo 2002 }\end{array}$ \\
Pós-graduação & Freqüência & $\%$ \\
Masculino & 6 & 60 \\
Feminino & 4 & 40 \\
Total & 10 & 100 \\
\hline
\end{tabular}

\begin{tabular}{|lcc|}
\hline \multicolumn{3}{|c|}{$\begin{array}{c}\text { Tabela 10. Distribuição segundo a área de pós-graduação, de } \\
\text { administradores de clínicas oftalmológicas. São Paulo 2002 }\end{array}$} \\
Pós-graduação & Freqüência & $\%$ \\
Administração hospitalar & 4 & 40 \\
Marketing & 2 & 20 \\
MBA & 3 & 30 \\
Recursos humanos & 1 & 10 \\
Total & 10 & 100 \\
\hline
\end{tabular}

Tabela 11. Distribuição das áreas de interesse para desenvolvimento profissional, de administradores de clínicas oftalmológicas. São Paulo 2002

\begin{tabular}{lcc|} 
Áreas pretendidas & Freqüência* & $\%$ \\
Informática & 6 & 17 \\
Recursos humanos & 4 & 11 \\
Jurídica & 6 & 17 \\
Financeira & 8 & 24 \\
Auditoria & 8 & 24 \\
Outras & 2 & 7 \\
Total & 34 & 100 \\
${ }^{*}$ Cada profissional poderia escolher uma ou mais áreas & \\
\hline
\end{tabular}

$\left.\begin{array}{|lcc|}\hline \text { Tabela 12. Participação societária do administrador de clínica } \\ \text { oftalmológica. São Paulo } 2002\end{array}\right)$

\begin{tabular}{|lcc|}
\hline \multicolumn{2}{|c|}{ Tabela 13. Distribuição dos administradores-sócios quanto à } \\
formação médica, em clínicas oftalmológicas de São Paulo 2002 \\
Sócio médico & Freqüência & $\%$ \\
Sim & 1 & 50 \\
Não & 1 & 50 \\
Total & 2 & 100 \\
\hline
\end{tabular}

Tabela 14. Distribuição de clínicas oftalmológicas pesquisadas, por sorteio, segundo o número de consultas/mês. São Paulo 2002

\begin{tabular}{lccc} 
Consultas/mês & Freqüência & $\%$ & $\%$ Acumulado \\
Até 999 & 7 & 46 & 46 \\
$1000-3999$ & 4 & 27 & 73 \\
$4000-7999$ & 3 & 20 & 93 \\
Acima de 8000 & 1 & 7 & 100 \\
Total & $15^{*}$ & 100 & \\
${ }^{\star} 5$ não informaram & & & \\
\hline
\end{tabular}




\begin{tabular}{|c|c|c|c|}
\hline Horas dedicadas & Freqüência & $\%$ & $\%$ Acumulado \\
\hline $20-39$ horas & 3 & 25 & 25 \\
\hline $40-59$ horas & 7 & 58 & 83 \\
\hline Acima de 60 horas & 2 & 17 & 100 \\
\hline Total & $12^{*}$ & 100 & \\
\hline
\end{tabular}

\begin{tabular}{|lcc|}
\hline \multicolumn{3}{|c|}{$\begin{array}{l}\text { Tabela 16. Distribuição, segundo o recebimento de benefícios de } \\
\text { administradores de clínicas oftalmológicas em São Paulo 2002 }\end{array}$} \\
Benefícios & Freqüência & $\%$ \\
Sim & 15 & 75 \\
Não & 5 & 25 \\
Total & 20 & 100 \\
\hline
\end{tabular}

\begin{tabular}{|c|c|c|}
\hline Benefícios & Freqüência & $\%$ \\
\hline Carteira assinada & 10 & 38 \\
\hline Plano de saúde & 12 & 44 \\
\hline Seguros & 5 & 18 \\
\hline Total & $27^{*}$ & 100 \\
\hline
\end{tabular}

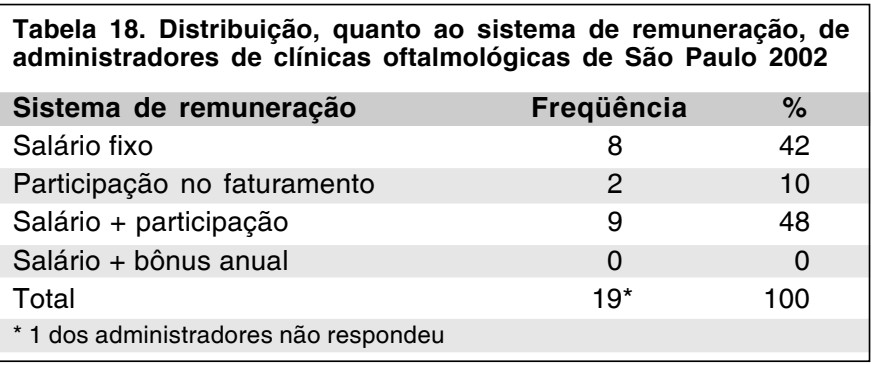

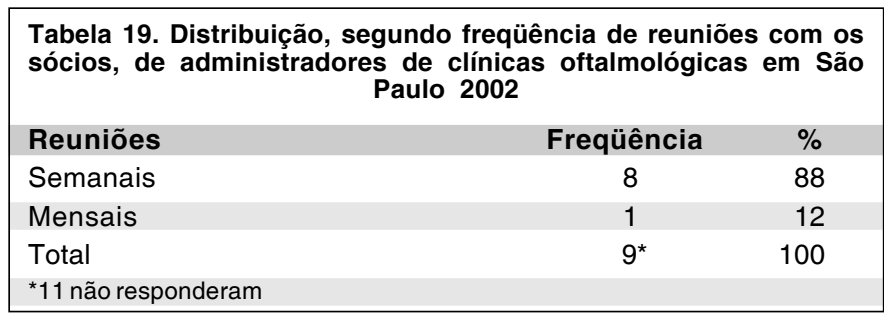

A área administrativa representa 70\% dos cursos de pósgraduação, com títulos específicos em Administração e Masters'in Business Administration (MBA). Avaliando-se médicos em funções gerenciais verificou-se que $20,7 \%$ tinham MBA ou equivalente ${ }^{(6)}$. Mezomo encontrou $60 \%$ de administradores hospitalares com pós-graduação em Administração(5).

\begin{tabular}{|c|c|c|}
\hline Principal responsável & Freqüência & $\%$ \\
\hline A empresa & 9 & 56 \\
\hline Empresa = administrador & 2 & 13 \\
\hline O administrador & 5 & 31 \\
\hline Total & $16^{*}$ & 100 \\
\hline
\end{tabular}

\begin{tabular}{lcc}
\hline \multicolumn{3}{l}{ Tabela 21. Distribuição segundo participação atual em cursos, de } \\
administradores de clínicas oftalmológicas em São Paulo & $\mathbf{2 0 0 2}$ \\
Participação atual em cursos & Freqüência & $\%$ \\
Sim & 8 & 40 \\
Não & 12 & 60 \\
Total & 20 & 100
\end{tabular}

As áreas apontadas como lacunas na formação profissional foram as de Finanças e Auditoria, seguidas pelas áreas de Informática e Jurídica. Foi encontrado maior interesse futuro nas áreas de Qualidade, Informática, Benchmarking e Planejamento Estratégico(6).

Somente dois administradores eram sócios da clínica e apenas um era médico. Uma justificativa seria formação deficiente para administrar, mesmo na área de saúde. Mezano detectou interesse em cursos na área administrativa em $78,6 \%$ dos médicos que exerciam administração hospitalar ${ }^{(5)}$.

Recentemente nota-se um aumento progressivo de mercado e salários, nos EUA, para os profissionais executivos na área de saúde ${ }^{(7)}$. Provavelmente o médico oftalmologista ainda não se interesse em ser administrador, mas não levantamos qual seria a razão.

Recomenda-se que estudantes de medicina, residentes e médicos devem receber cursos sobre Administração em Medi$\operatorname{cina}^{(8)}$. Por outro lado, registra-se progressivo aumento no número de cursos, na área de administração em medicina, surgindo nos EUA ${ }^{(8)}$.

Clínicas de portes variados, com menos de 1.000 consultas/ mês (46\%) até uma clínica que tem mais de 8.000 consultas /mês utiliza-se de serviços profissionais específicos para sua administração. Isso mostra a grande necessidade de profissionais na área. Nas duas clínicas administradas por médicos, uma tinha o porte de 300 consultas /mês e a outra 6.000 consultas/mês, esta administrada por um dos sócios, após experiência não bem sucedida com outros administradores, não médicos.

O número de horas dedicadas efetivamente à administração foi alto, demonstrando que a atividade consome muitas horas semanais; $75 \%$ dos administradores trabalham mais de 40 horas/semana. Mezomo (1987) encontrou 52\% de administradores trabalhando uma média de $9-15 \mathrm{~h} / \mathrm{dia}^{(5)}$. Foi demonstrado que médicos em funções executivas trabalham uma média de 50 horas semanais( ${ }^{(6)}$.

Os benefícios foram freqüentes para $75 \%$ dos administra- 
dores. Alguns administradores receberam mais de um benefício. Predominou o plano de saúde com $44 \%$, seguido de carteira assinada com $38 \%$.

Houve ligeiro predomínio do salário com participação no faturamento da clínica (48\%), seguido de salário fixo para $42 \%$ dos administradores.

Em relação à freqüência de reuniões com os sócios, tivemos pouco retorno nos questionários. Alguns administradores comentaram que era muito "variável”. Em geral tentavam resolver diretamente com os sócios, à medida que os problemas surgissem.

A maioria dos administradores (56\%) atribui à clínica a responsabilidade pelos investimentos e direcionamento na carreira. Atualmente $60 \%$ dos administradores não participam de cursos na área.

A dificuldade para discussão maior encontra-se no fato de não encontrarmos na literatura trabalhos com o mesmo foco (administração de clínicas oftalmológicas), a fim de estabelecermos comparações.

\section{CONCLUSÕES}

Neste estudo, realizado entre administradores de clínicas oftalmológicas da Grande São Paulo, em 2002, não houve predominância de sexo, a idade mais encontrada na faixa de 30 a 39 anos e geralmente, não é sócio do grupo. Sua profissão: geralmente não é médico e tem formação (graduação) em Administração de Empresas. Metade desses profissionais fez pós-graduação, principalmente em Administração e MBA. A administração de clínica oftalmológica foi a primeira experiência do tipo para $60 \%$ dos administradores. Os benefícios principais do cargo são carteira assinada e plano de saúde. A carga horária de trabalho é superior a 40 horas semanais. $\mathrm{Na}$ sua visão de carreira, sente-se dependente de iniciativa maior da empresa (clínica oftalmológica) para seu aprimoramento profissional. A sistemática de remuneração predominante é o salário com participação nos resultados.

É uma profissão nova, que está se estruturando, e há necessidade de que outros estudos sejam feitos para se fazer comparações e termos uma visão melhor do quadro profissional na área.

\section{ABSTRACT}

Purpose: To check the profile of the ophthalmology clinic administrator in Great São Paulo. Methods: Several ques- tionnaires were sent to clinics at random. Sex, age, schooling, graduate and postgraduate courses, partnership in the clinic, working hours, career plan, payment system and the amount of appointments per month in the clinic. Results: $55 \%$ of them were men, and $60 \%$ of them were 30 to 59 years old. For $60 \%$ of them, this was their first experience in the area. 56\% were in charge of clinics with more than 1,000 appointments per month. Among those with college degrees (70\%), 56\% had a degree in Business Administration. Half of the researched people had post-graduate courses, and the most frequent were Hospital Administration (40\%) and MBA (30\%). Only 10\% were partners in the clinic. $75 \%$ work more than 40 hours per week. At present $60 \%$ are not attending specific courses and $56 \%$ think that it is the clinic's responsibility to incentive their careers. Benefits: $75 \%$ have private health insurance and/or are registered workers. Earning: salary with participation in the clinic's income (48\%) and a fixed salary (42\%). Conclusions: In this study we found the profession of administrator of clinics being structured, with the participation of male and female professionals, graduated in Business Administration, some with postgraduate courses on a related subject, working over 40 hours per week, thinking that the clinic should invest in their professional growth. Further research is required for a better understanding of this professional.

Keywords: Practice management, medical/trends; Office management/trends; Organizational case studies; Physician executives; Practice management/trends; Professional practice; Optimization

\section{REFERÊNCIAS}

1. Fayol H. Administração industrial e geral. 7a ed. São Paulo: Atlas; 1968.

2. Mintzberg H.The manager's job: folklore and fact. Harvard Business Rev 1975;53:46-61.

3. Martins PEM. O desafio de formar administradores para o Brasil do terceiro milênio. In: Martins PEM. Recursos humanos: foco na modernidade. Rio de Janeiro: Qualitymark; 1992. p.237.

4. Andrade N. Suporte administrativo de clínica oftalmológica. In: Centurion V. As bases da administração em oftalmologia. Rio de Janeiro: Cultura Médica; 2001. p.60.

5. Mezomo JC. O administrador hospitalar dos hospitais de São Paulo em 1986. Hosp Adm Saúde 1987;11:112-6.

6. Williams SJ. Training needs for physician leaders. J Health Adm Educ 2001;19:195-202.

7. Kirchheimer B. Reaping the rewards. Hospitals show their appreciation for top execs by boosting compensation. Mod Healthc 2001;31:27-30,32-3,38.

8. Backer LA. Back to school: options for learning the business of medicine. Fam Pract Manag 2001;8:27-33. 